\title{
Fentanyl Overdose Causing Hippocampal Ischaemia Followed by Delayed Leukoencephalopathy
}

\author{
Aaron R. Switzer, Benjamin Beland, Justyna R. Sarna, Alison Walzak, \\ Gerald Pfeffer (iD
}

Keywords: Cognitive Impairment, Magnetic Resonance Imaging, Fentanyl Overdose, Toxic Leukoencephalopathy, Ischemic Brain Injury

doi:10.1017/cjn.2020.33

Can J Neurol Sci. 2020; 47: 398-399

A 52-year-old man developed acute amnestic syndrome after being found unconscious for over 12 hours. After roughly 24 hours, his delirium had cleared and his neurological examination was normal except for memory deficits. His Mini-Mental State Exam score was 18/30, due to disorientation, attentional deficit and delayed recall impairment. Urine drug screen was positive for fentanyl. Brain magnetic resonance imaging (MRI) revealed restricted diffusion in both hippocampi with associated T2 FLAIR hyperintensities (Figure 1). His hippocampal injuries were thought to be due to a direct toxic effect of fentanyl and from hypoxaemia secondary to opioid-related hypoventilation. ${ }^{1} \mathrm{He}$ received supportive management, and workup for other potential aetiologies with bloodwork and electroencephalogram were unremarkable.

Over the next month, the patient developed rigidity, rest tremor and a shuffling gait. Follow-up MRI demonstrated diffuse leukoencephalopathy (Figure 2A). He was started on a trial of levodopa-carbidopa. At 5-month follow-up, his parkinsonism had improved where only his resting tremor remained. Neuropsychology testing showed minimal recognition memory, poor volition, impaired attention with preserved general knowledge and reasoning. MRI at 5-month follow-up showed significant improvement of diffuse leukoencephalopathy (Figure 2B). When last seen, he was still admitted to the hospital awaiting placement in a long-term care facility.

Isolated bilateral hippocampal lesions are a rare neuroimaging finding that has characteristically been associated with toxic aetiologies such as opioids, cocaine or carbon monoxide. ${ }^{2}$ This neuroimaging finding has recently been described in a cluster of cases in the context of fentanyl overdose. ${ }^{1}$ Familiarity with this relationship is important given the ongoing opioid crisis in Canada, primarily driven by the use of fentanyl from both prescription and illegal sources. ${ }^{3}$ The predilection for injury to the hippocampi in opioid use is supported by reversible hypoxia/ hypoglycaemia animal models demonstrating worse recovery to this brain region with opioid use. ${ }^{4}$ Peculiarly, another reported reversible hypoxia/hypoglycaemia model comparing various opioid types demonstrated a negative effect of morphine but not fentanyl, ${ }^{5}$ which is contradicted by the clinical literature in humans showing this can occur with various opioids among other substances. ${ }^{6}$ It is also possible that an interaction of various

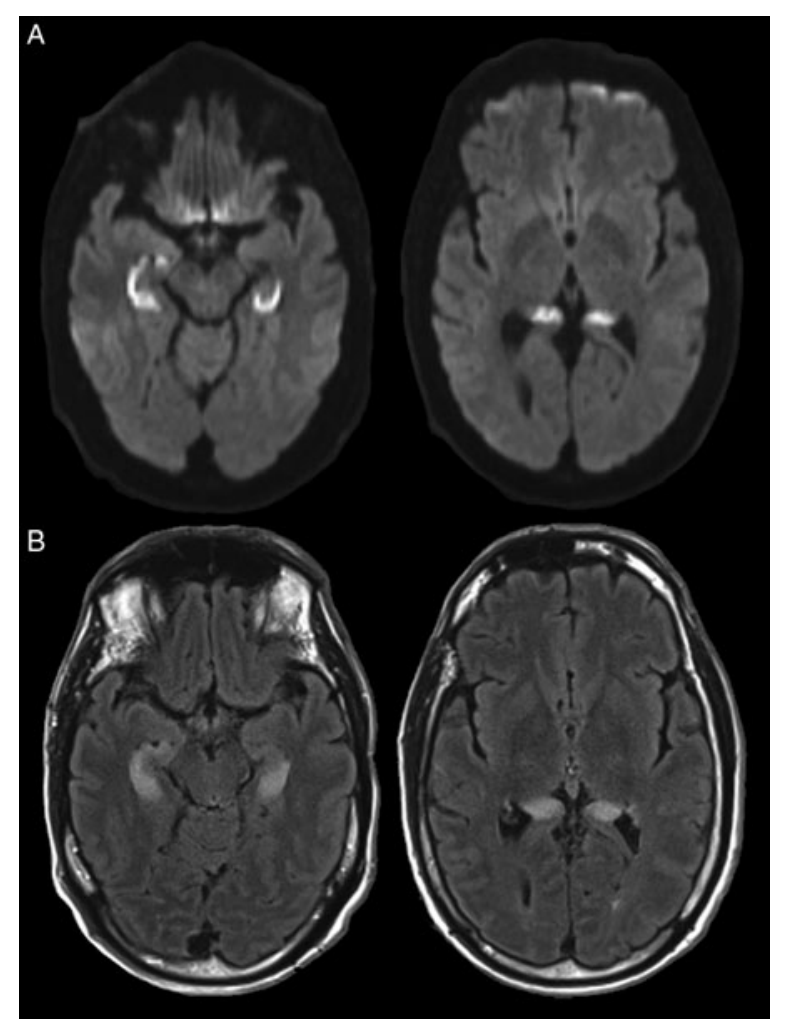

Figure 1: MRI brain without contrast. Brain MRI showed (A) restricted diffusion on diffusion-weighted images and (B) T2 FLAIR hyperintensities in the hippocampi bilaterally.

From the Department of Clinical Neurosciences, Cumming School of Medicine, University of Calgary, Calgary, AB, Canada (ARS, BB, JRS, GP); Department of Medicine, Cumming School of Medicine, University of Calgary, Calgary, AB, Canada (AW); Hotchkiss Brain Institute, University of Calgary, Calgary, AB, Canada (JRS, GP); Alberta Child Health Research Institute, University of Calgary, Calgary, AB, Canada (GP)

Received November 29, 2019. Final Revisions Submitted January 23, 2020. Date of Acceptance February 10, 2020.

Correspondence to: Gerald Pfeffer, Department of Clinical Neurosciences, University of Calgary, HMRB 155, 3330 Hospital Dr NW, Calgary, AB, T2N 4N1, Canada. Email: gerald.pfeffer@ucalgary.ca 


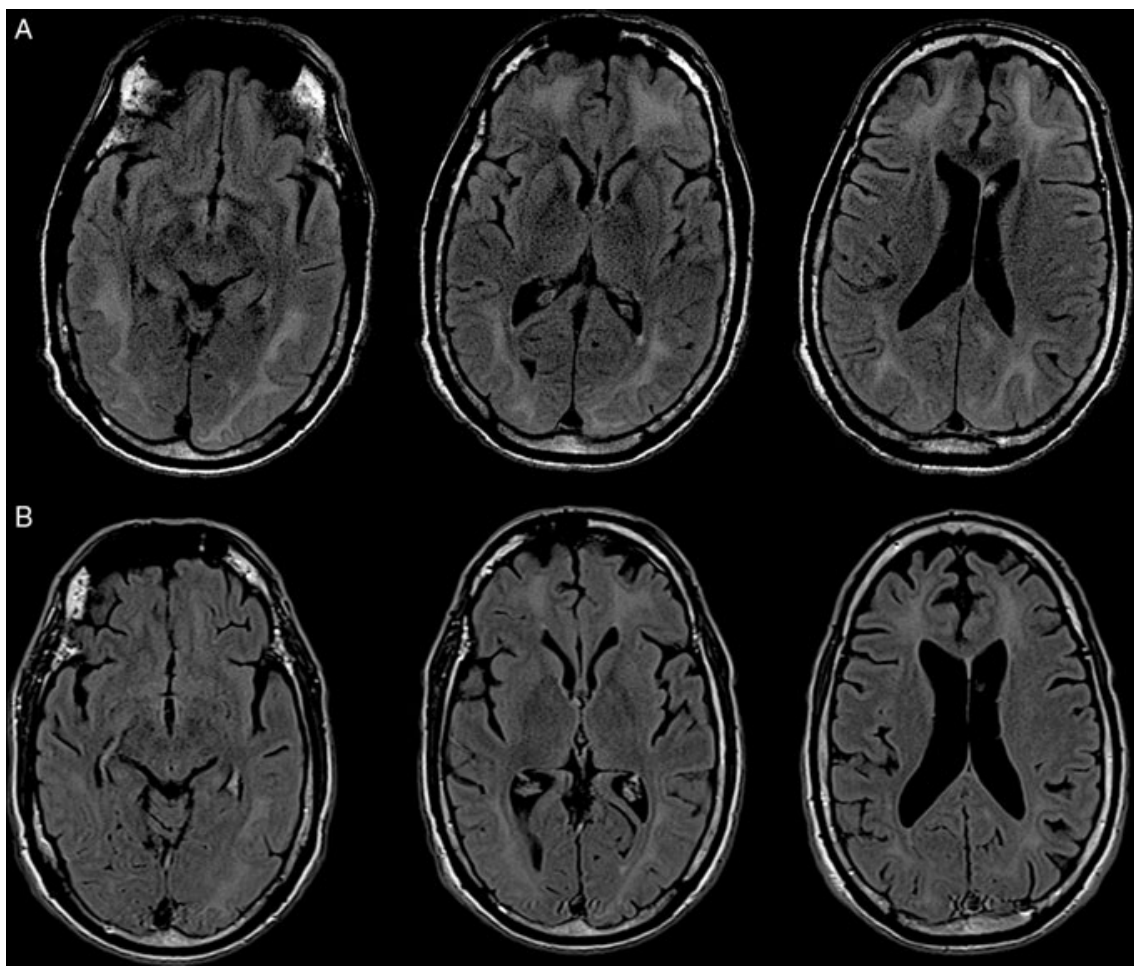

Figure 2: Follow-up brain MRI without contrast. (A) T2 FLAIR images showed diffuse white matter hyperintensities at 1-month follow-up. (B) T2 FLAIR images at 5-month follow-up showed significant improvement of white matter hyperintensities.

substances increases the likelihood of this complication, as has been suggested by numerous reports. ${ }^{6-8}$

A unique feature of our case is the availability of follow-up imaging that demonstrated a delayed-onset diffuse leukoencephalopathy with partial improvement at 5-month follow-up imaging. We are not aware of any prior case demonstrating the progression of findings from isolated hippocampal ischaemia to diffuse leukoencephalopathy, in the context of fentanyl overdose. Prior reports demonstrate similarities in the clinical findings and neuroimaging features of delayed leukoencephalopathy in the context of substance abuse..$^{8-10}$ Our report brings attention to this unique presentation and neuroimaging findings of fentanyl overdose associated with an acute amnestic syndrome and selective hippocampal ischaemia that progresses to a delayed-onset leukoencephalopathy.

\section{CONFLict of InTEREST}

This was not a funded study. Dr. JRS reports personal fees from Abbvie, personal fees from Allergan, outside the submitted work. The other authors report no competing interests.

\section{Statement of Authorship}

ARS was primary author of the manuscript, analysed clinical data and conceptualised the study. BB, JRS and AW analysed clinical data and revised the manuscript for intellectual content. GP conceptualised the study, analysed clinical data and authored the manuscript.

\section{REFERENCES}

1. Barash JA, Ganetsky M, Boyle KL, et al. Acute amnestic syndrome associated with fentanyl overdose. N Engl J Med. 2018;378: 1157-8.

2. Barash JA, Kofke WA. Connecting the dots: an association between opioids and acute hippocampal injury. Neurocase 2018;24:124-31.

3. Belzak L, Halverson J. The opioid crisis in Canada: a national perspective. Health Promotion Chronic Dis Preven Canada 2018;38:224-33.

4. Ammon-Treiber S, Stolze D, Schröder H, Loh H, Höllt V. Effects of opioid antagonists and morphine in a hippocampal hypoxia/hypoglycemia model. Neuropharmacology 2005;49: 1160-9.

5. Ammon-Treiber S, Stolze D, Höllt V. Differential effects of muopioid receptor agonists in a hippocampal hypoxia/hypoglycemia model. Brain Res. 2007;1183:60-5.

6. Barash JA, Somerville N, DeMaria A. Cluster of an unusual amnestic syndrome - Massachusetts, 2012-2016. MMWR Morb Mortal Wkly Rep. 2017;66:76-9.

7. Duru UB, Pawar G, Barash JA, Miller LE, Thiruselvam IK, Haut MW. An unusual amnestic syndrome associated with combined fentanyl and cocaine use. Ann Intern Med. 2018; 169:662-3.

8. Kesari NK, Fleet T, Alenzi B, Goodman JC. Mystery case: a 48-year-old woman with bizarre behavior, neurologic symptoms, and progressive decline. Neurology 2018;90:242.

9. Barnett MH, Miller LA, Reddel SW, Davies L. Reversible delayed leukoencephalopathy following intravenous heroin overdose. J Clin Neurosci. 2001;8:165-7.

10. Zanin A, Masiero S, Severino MS, Calderone M, Da Dalt L, Laverda AM. A delayed methadone encephalopathy: clinical and neuroradiological findings. J. Child Neurol. 2009;25: $748-51$. 